\title{
A cuckoo search based neural network to predict fatigue life in rotor blade composites
}

\author{
Khaled Ziane ${ }^{1 *}$, Adrian llinca ${ }^{1}$, Abdullah Khan ${ }^{2}$ and Soraya Zebirate ${ }^{3}$ \\ 1 Wind Energy Research Laboratory (WERL), Université du Québec à Rimouski, 300, allée des Ursulines, Rimouski, G5L 3A1, CANADA \\ Phone: +1-418-732-7232 \\ ${ }^{2}$ Institute of Business and Management Science, University of Agricultural Peshawar, PAKISTAN \\ ${ }^{3}$ Laboratoire d'Ingénierie en Sécurité Industrielle et Développement Durable LISIDD, IMSI, Université d'Oran 2 Mohamed Ben Ahmed, ALGERIA
}

\begin{abstract}
In modern wind turbine blades industry, fiber-reinforced composites are mostly used for their good mechanical characteristics: high stiffness, low density and long fatigue life. Wind turbine blades are constructed in different structural elements from a variety of composite laminates, often including Unidirectional (UD) material in spars and multiple forms of Multidirectional (MD) in skins and webs. The purpose of this paper is to identify materials that have appropriate fiber orientations to improve fatigue life. By using Cuckoo Search-based Neural Network (CSNN), we have developed a model to predict fatigue life under tension-tension charges for five composite materials, with different fiber stacking sequences embedded in three types of resin matrices (epoxy, polyester and vinylester), which are all appropriate for the design of wind turbine blades. In the CSNN approach used in this work, the cost function was assessed using the Mean Square Error (MSE) computed as the squared difference between the predicted values and the target values for a number of training set samples, obtained from an experimental fatigue database. The results illustrate that the CSNN can provide accurate fatigue life prediction for different MD/UD composite laminates, under different angles of fiber orientation.
\end{abstract}

ARTICLE HISTORY

Revised: $4^{\text {th }}$ Nov 2019

Accepted: $4^{\text {th }}$ Nov 2019

\section{KEYWORDS}

Wind turbine blades; composite materials; fiber orientation; fatigue life prediction; cuckoo search; neural network.

\section{INTRODUCTION}

As the blade is the most important component within wind turbines in capturing energy, it must be designed structurally strong to support the different loads to which it may be exposed. The design of the wind turbine depends heavily on the materials of the blade, which must have a high stiffness, low density and long fatigue life properties [1]. The class of materials that have met these requirements best has been fiber-reinforced composites using mostly glass or carbon. The Glass Fiber-Reinforced Plastic (GFRP) composites are generally characterized by good static and fatigue mechanical properties combined with low-cost components and fabrication processes [2], which can extend the lifetime of the turbine blades.

Wind turbine blades are typically made from E-glass fibers (for its high electric resistance), which are used as main reinforcement in the composites [3]. A low-cost glass fiber grade combining high strength (2 GPa) and moderate stiffness $(76 \mathrm{GPa})$ are used together with a thermoset polymer matrix such as epoxy, polyester or vinylester [3]. Sandwich constructions, consisting of multiaxial composite laminates, together with balsa wood or Polyvinyl Chloride (PVC) foam, are used for the outer shell and for the shear webs in the blades, as shown in Figure 1.

The wind turbine blades are manufactured in separate structural components using a range of composite laminate kinds, often including Unidirectional (UD) material in spars and several forms of Multidirectional (MD) reinforcement, such as biaxial and triaxial fabrics in skins and webs (see Figure 1). Depending on the component fibers, resin, type and direction of loading, each may have a substantial sensitivity to fatigue [5]. 


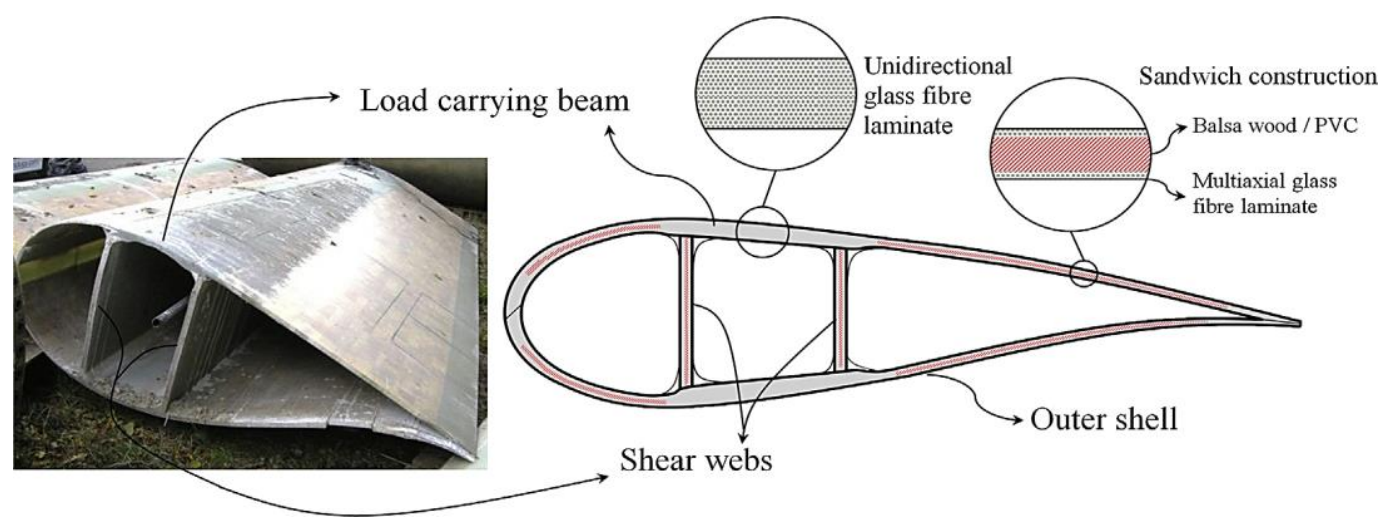

Figure 1. Typical cross-section of rotor blade [4].

To determine safety factors more precisely, detailed information about the effects of different loading conditions (extreme winds, moisture, temperature variations, etc.) and the microstructure parameters of wind blade composites (such as fiber sizing, orientation, nanoscale-engineered matrix, etc.) on their strength and lifetime is required [3]. The lifetime and strength prediction analysis of these parameters are among the important tasks to improve the reliability and performance of the wind turbine rotor blades. In addition, predicting the fatigue conduct of these subcomponents could be the first step towards anticipating the general conduct of laminates under cyclic loading, which can be considered as a way of preventing the failure of our blades. In this perspective, Artificial Neural Networks (ANN) are a very common tool for applications that require data processing for predictive purposes, due to their massively parallel structure. ANN can also deal with many multivariable nonlinear problems for which an accurate analytical solution is difficult to obtain [6].

One of the most common neural network architectures is Feedforward Neural Network (FNN), which has been mainly used due to their well-known universal approximation capabilities. Feedforward means that the signal (dataset) flows in one direction, from the input to the output layer (forward) through one or more hidden layers. This topology allows inserting and combining some types of algorithms in weight training instead of the Backpropagation (BP) algorithm and Levenberg Marquardt (LM), which are integrated by default in certain software; these are gradient-based techniques. Currently, there have been many algorithms used to learn and optimize the FNN, among them we can mention: Genetic Algorithms (GA), Particle Swarm Optimization (PSO), Cuckoo Search algorithms (CS), and so on.

The power of almost all modern metaheuristics comes from the fact that they imitate the best feature in nature, especially biological systems evolved from natural selection over millions of years [7]. CS is one of the latest natureinspired metaheuristic algorithms proposed by Xin-Shen Yang [7] for solving optimization problems. CS is based on the brood parasitism of some cuckoo species. In addition, this algorithm is enhanced by the so-called Lévy flights, rather than by simple isotropic random walks [8]. Recent studies show that CS is potentially far more efficient than PSO and GA [8]. In particular, CS can be modified to provide a relatively high convergence rate to the true global minimum [9].

This work aims to use this new combination (cuckoo search-based neural network CSNN) in the training of SNL/MSU database [10], which was developed by the Sandia National Laboratories (SNL) in collaboration with Montana State University (MSU). The dataset we used is collected from their large public database, for the purpose of performing comparative analysis between unidirectional and multidirectional materials, in terms of fatigue life. The studied materials are all intended for the design of wind turbine blades. The GFRP composites from E-glass fibers and thermoset polymers were the most suitable choice for our analysis, because of their use by the most of wind turbine manufacturers, owners and contractors.

This database is the latest of a series of tests that the SNL have been publishing over the years. The novelty of our work is that it applies CSNN on this newly published data and creates a model that is capable of predicting the fatigue life of different MD/UD composite materials. This model could be used in the future as an alternative to the costly lab tests, as well as to validate new experimental results.

The materials of wind turbine blades and their fatigue tests have attracted attention of many researchers and the academic community in general. However, our research and literature review did not find any studies about the application of artificial intelligence methods on the prediction of fatigue behavior of wind turbine blades materials. The most representative results of using Google search engine with the keywords "artificial intelligence in fatigue life prediction of wind turbine materials" are the works we published earlier [11, 12]. In what follows, we will discuss some studies relevant to this work.

Artificial neural networks (ANN) have been previously used by Ziane et al. [11,12] to predict the fatigue strength of different composite materials, intended for the design of wind turbine blades, under tension-tension and compressioncompression loadings. A backpropagation and particle swarm optimization were used as training algorithms. They had reached the conclusion that the size of the database represents a challenge to the traditional regression, hence the need for an artificial intelligence method that models the problem. Indeed, this work is based on a portion of their data used with the PSO [11], in order to improve the results already found. 
Al-Assadi et al. [6, 13] stated that ANN have already been used in modeling the static and cyclic behavior of composites, controlling the manufacturing parameters used in their machining and/or predicting their wear properties. These ANN offer an accurate and cost-effective approach for modeling fatigue life, and are employed as a black box rendering the knowledge of its internal behavior unnecessary to most users.

Carbon fiber and glass fiber-reinforced composites have been used by Mathur et al. [14] to evaluate the performance of ANN in predicting the fatigue lives of laminates under different lay-up. They showed that the prediction of fatigue lives of composite material is affected by various factors such as stress amplitude, stress ratio and statistical scatter of fatigue life data. Another advantage was that ANN could be used as efficient tool in predicting the fatigue life of composite material when complete data sets are not available.

Jiménez et al. [15] applied artificial neural networks and Weighted K-Nearest Neighbors WKNN as pattern recognition approaches, for detecting and diagnosing the delamination in wind turbine blades. This common structural problem can cause cracks, and partial or total breakage of the blade. They concluded that the performance of diagnostic system testing for multi-level detection of delamination in ANN and WKNN classifiers indicate a high level of accuracy; according to them, ANN was the best classifier.

Recently, Liu et al. [16] presented a paper on the application of PSO-BP neural network for strain prediction of wind turbine blades. They have found that this new approach can be utilized to predict the strain of unmeasured points of wind turbine blades during static testing, and this provides more data for characteristic structural parameters calculation. They have suggested that the outputs can also be used as inputs for another process for life prediction of wind blades.

\section{EXPERIMENTAL FATIGUE DATA}

Wind turbine blades are designed to several major structural conditions, including tip deflection, strength and buckling during severe loading, as well as very high numbers of fatigue cycles and various service environments [17]. Sandia National Laboratories (SNL) performed the experimental stage of this paper with wind turbine-related materials and posted it on the laboratory's website [10]. Moreover, a wide range of prospective blade materials have been included in the course of this work, including E-glass-fiber/polyester, vinylester and epoxy resins in the form of a variety of laminate structures, fiber contents and numerous stitched fabrics.

The types and sources of resins used during the manufacturing process are listed in Table 1. All materials were handled with the resin infusion method in closed molds that were shaped without machining into their final dogbone form. The nomenclature of laminates corresponds to the Sandia National Laboratories. Consequently, the specified fabric details show the stitching and transverse strands or mat to which the main strands are stitched. Laminates were treated by infusion through layers of resin distribution [18, 19]; a more comprehensive description of the manufacturing method can be found in $[11,20]$.

Table 1. MD/UD materials investigated.

\begin{tabular}{|c|c|c|c|c|c|c|c|c|}
\hline \multicolumn{3}{|c|}{ MD/UD LAMINATES } & \multicolumn{6}{|c|}{ MATRIX MATERIALS } \\
\hline $\begin{array}{l}\text { General } \\
\text { material } \\
\text { name }\end{array}$ & Lay-up & $\begin{array}{c}\text { Fiber } \\
\text { volume } \\
\text { fraction }(\%)\end{array}$ & Type & & \multicolumn{2}{|c|}{ Resin } & \multicolumn{2}{|c|}{$\begin{array}{c}\text { Cure and } \\
\text { post cure } \\
\text { temperature } \\
\left({ }^{\circ} \mathrm{C}\right) \\
\end{array}$} \\
\hline $\begin{array}{l}\text { PPG1200- } \\
\text { EP1 }\end{array}$ & $\begin{array}{c}{\left[\left( \pm 45^{\circ}\right)_{2} /(0)_{2}\right]_{s}} \\
{[0]_{2}}\end{array}$ & $\begin{array}{c}58 \\
56-61\end{array}$ & Epoxy & \multicolumn{3}{|c|}{$\begin{array}{c}\text { Hexion MGS } \\
\text { RIMR135/RIMH1366 }\end{array}$} & \multicolumn{2}{|c|}{20 and 90} \\
\hline $\begin{array}{l}\text { PPG1200- } \\
\quad \text { UP5 }\end{array}$ & $\begin{array}{c}\left.\left( \pm 45^{\circ}\right)_{2} /(0)_{2}\right]_{s} \\
{[0]_{2}}\end{array}$ & $\begin{array}{l}58 \\
58\end{array}$ & Polyester & \multicolumn{3}{|c|}{$\begin{array}{c}\text { Reichhold Polylite } \\
\text { X4627-31 with 2\% } \\
\text { MCP-75FRED }\end{array}$} & \multicolumn{2}{|c|}{25 and 70} \\
\hline $\begin{array}{l}\text { PPG1200- } \\
\text { VE4 }\end{array}$ & $\begin{array}{c}\left.\left( \pm 45^{\circ}\right)_{2} /(0)_{2}\right]_{s} \\
{[0]_{2}}\end{array}$ & $\begin{array}{l}58 \\
58\end{array}$ & Vinylester & \multicolumn{3}{|c|}{$\begin{array}{l}\text { Reichhold Dion } \\
\text { X4486-14 }\end{array}$} & \multicolumn{2}{|c|}{25 and 70} \\
\hline $\begin{array}{l}\text { PPG1200- } \\
\text { VE5 }\end{array}$ & $\begin{array}{c}\left.\left( \pm 45^{\circ}\right)_{2} /(0)_{2}\right]_{s} \\
{[0]_{2}}\end{array}$ & $\begin{array}{l}59 \\
57\end{array}$ & Vinylester & \multicolumn{3}{|c|}{$\begin{array}{l}\text { Reichhold Dion } \\
\text { X4235-91 }\end{array}$} & \multicolumn{2}{|c|}{35 and 70} \\
\hline $\begin{array}{l}\text { PPG1200- } \\
\text { VE6 }\end{array}$ & $\begin{array}{c}\left.\left( \pm 45^{\circ}\right)_{2} /(0)_{2}\right]_{s} \\
{[0]_{2}}\end{array}$ & $\begin{array}{l}60 \\
58\end{array}$ & Vinylester & \multicolumn{3}{|c|}{$\begin{array}{l}\text { Reichhold Dion } \\
\text { X4627-39 }\end{array}$} & \multicolumn{2}{|c|}{25 and 70} \\
\hline \multicolumn{9}{|c|}{ Glass fabric specifications } \\
\hline \multirow{2}{*}{$\begin{array}{c}\text { Fabric } \\
\text { Manufacturer }\end{array}$} & \multirow{2}{*}{\multicolumn{2}{|c|}{ Designation }} & \multirow{2}{*}{$\begin{array}{l}\text { Areal weight } \\
\qquad\left(\mathrm{g} / \mathrm{m}^{2}\right)\end{array}$} & \multicolumn{5}{|c|}{$\begin{array}{c}\text { Component strands warp direction } \\
\text { (Weight \%) }\end{array}$} \\
\hline & & & & $0^{\circ}$ & $\pm 45^{\circ}$ & $90^{\circ}$ & Mat & Stitch \\
\hline \multirow{2}{*}{$\begin{array}{l}\text { PPG-Devold } \\
\text { LLC }\end{array}$} & $\mathrm{L} 1200 /$ & $50-\mathrm{E} 07$ & 1261 & 91 & 0 & 4 & 4 & 1 \\
\hline & DB810 & E05-A & 808 & 0 & 99 & 0 & 0 & 1 \\
\hline
\end{tabular}


Standard laminates discussed in this paper include the following reinforcement structures:

- Unidirectional stitched fabrics where primary strands at $0^{\circ}$ are aligned and stitched to a backing including transverse strands and/or mat.

- Multidirectional laminates reinforced with 'triax' fabrics having layers at $0^{\circ}$ and $\pm 45^{\circ}$.

Fatigue Parameters and Properties

Fatigue experiments are carried out under load control at varying maximum loads and for different $R$-values, where $R$ is the ratio of minimum to maximum load, resulting in typical data sets of stress or strain versus cycles of failure $(S-$ $N$ ), generally curve fit to power law $[2,5]$ :

$$
\begin{aligned}
& S=A \times N^{B} \\
& N=C \times S^{b}
\end{aligned}
$$

where $S$ is the maximum stress or strain, $N$ is the number of cycles to failure, $A$ and $C$ are constants, $B$ and $b$ are fatigue exponents, with $B=1 / b$. The form and notation in Equation. (1) is used to represent data in this paper. The fatigue exponent may also be represented as $n$, where:

$$
n=-1 / B
$$

While the power law model is generally preferred, exponential and three-parameter models have also been used for blade materials; the power law representation has been shown to provide a good fit to $S-N$ data for small strands up to $10^{10}$ cycles $[2,5]$.

Normalizing the stress by the static strength has the effect of reducing discrepancies caused by variations in fiber content and fraction of $0^{0}$ plies in MD laminates [5]. The use of strain has a similar effect without normalization. Strain curve fits (Equation. (1)) can differ from stress fits in terms of exponents, and reflect the nonlinearity of stress-strain response at higher stresses, particularly for biax and MD laminates. Strain is often impossible to monitor over the entire coupon lifetime due to instrumentation failure.

Table 2 presents a comparison of fatigue data trends, which included UD fabric and MD laminates, all based on the same strands and resins. Blades are usually constructed from most or all of these materials in which the adjacent materials must withstand similar strains. Comparison of fatigue exponents $B$ and $n$ (Equations. (1) and (3)) and the cycle fatigue strain in the order of millions gives some indication of the most critical areas of blades. The curve fit in Equation. (1) is normalized as a function of the static tensile strength and UTS (Ultimate Tensile Strength determined at the standard test rate of $0.0254 \mathrm{~mm} / \mathrm{s}$ ), by including A/UTS, which does not affect the exponent [5]. These properties must be viewed in the context of actual blade stress distributions and the potential presence of flaws.

Table 2. Summary of tensile strength and fatigue trends $(\boldsymbol{R}=\mathbf{0 . 1})$ for various blade materials.

\begin{tabular}{ccccccc}
\hline Material form & Resin & $\begin{array}{c}\text { UTS } \\
(\mathrm{MPa})\end{array}$ & A/UTS & $B$ & $n$ & $\begin{array}{c}10^{6} \text { cycle } \\
\text { strain }(\%)\end{array}$ \\
\hline \multicolumn{7}{c}{ MD Laminates, UD fabric L1200/G50-E07 and biax fabric DB810-E05-A } \\
\hline$\left[\left( \pm 45^{\circ}\right)_{2} /(0)_{2}\right]_{s}$ & EP-1 & 704 & 1.957 & -0.130 & 7.69 & 0.79 \\
{$\left[\left( \pm 45^{\circ}\right)_{2} /(0)_{2}\right]_{s}$} & VE-4 & 628 & 1.955 & -0.146 & 6.85 & 0.53 \\
{$\left[\left( \pm 45^{\circ}\right)_{2} /(0)_{2}\right]_{s}$} & UP-5 & 663 & 1.736 & -0.151 & 6.62 & 0.42 \\
\hline \multicolumn{7}{c}{ UD Fabric L1200/G50-E07 Laminates } \\
\hline$[0]_{2}$ & EP-1 & 995 & 1.265 & -0.088 & 11.4 & 0.88 \\
{$[0]_{2}$} & VE-4 & 912 & 2.485 & -0.170 & 5.88 & 0.53 \\
{$[0]_{2}$} & UP-5 & 884 & 1.940 & -0.173 & 5.78 & 0.39 \\
\hline
\end{tabular}

\section{INTELLIGENT PREDICTION METHODS}

A program in artificial intelligence has been modeled to see what our qualities are like species, as well as our associative trend [21]. If we want to model human intelligence, then it may be feasible through modeling individuals in a social context. Social behavior allows individuals of a species to adapt to their environment, especially providing them with more information than their sensory abilities can muster.

The numerical prediction consists of predicting values that characterize the input data. The most widely used method for numerical forecasting is the regression [21]. However, as we have seen above, some classification techniques (such as neural networks, cuckoo search, etc.) can be adapted for prediction. 


\section{Artificial Neural Networks}

Artificial neural networks (ANN) are networks of simple processing elements (called' neurons') that operate and communicate with other elements on their local data [22, 23, 24]. ANN's design was inspired by an actual brain system, but as shown in Figure 2, the processing elements and architectures used in ANN have gone far from their biological inspiration. $W_{i}$ is the synaptic weights matrix linking the input with the hidden layer, and $W_{o}$ is the synaptic weights matrix linking the hidden layer with the output.

In latest years, ANN has discovered its application for multiple purposes in the field of fatigue [22]. Depending on the network processing of data, they can be classified as feedforward or recurrent. They can be further categorized as supervised, unsupervised or reinforced ANN according to the learning rules [25].

In general, feedforward ANN consist of a layer of input neurons, a layer of output neurons and one or more layers of hidden neurons [26, 27]. These networks are usually fully connected, meaning that a neuron is connected to all the neurons in the previous layer. Every neuron in the output and hidden layers follows the general model of the neuron [6]. The input signal propagates layer-by-layer through the network in a forward direction. Different transfer functions such as sigmoidal, linear or triangular were used to model the activity of the neurons [13].

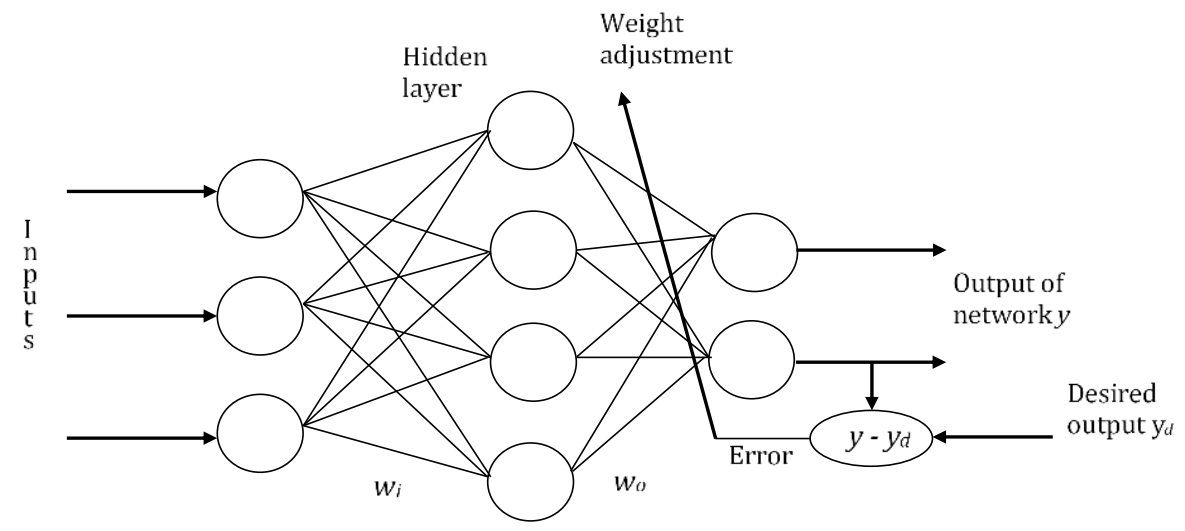

Figure 2. Feedforward neural network with one hidden layer.

The input to a node $i$ in the $k^{\text {th }}$ layer is given by Equation. (4):

$$
\operatorname{net}_{i, k}=\left[\sum_{j} w_{i, j, k} \text { out } t_{j, k-1}\right]+\theta_{i, k}
$$

where $w_{i, j, k}$ represents the weight connection strengths for node $j$ in the $(k-1)^{t h}$ layer to node $i$ in the $k^{t h}$ layer; out $_{j, k}$ is the output of node $i$ in the $k^{\text {th }}$ layer and $\theta_{i, k}$ is the threshold associated with node $i$ in the $k^{\text {th }}$ layer [26].

The backpropagation training algorithm (such as Levenberg-Marquardt) is frequently used to iteratively minimize the cost function as defined later in (Equation. (8)) with respect to the interconnection weights and neurons thresholds. However, the main drawback of this algorithm is that the solution could be trapped in local minima and might converge at slow speed. This disadvantage can be avoided by using cuckoo search training algorithm, which can solve the problem of local minima and achieve fast convergence $[8,9,28]$.

\section{Cuckoo Search Algorithm}

Cuckoo Search (CS) algorithm has been found to be very efficient in solving global optimization problems [7]. This algorithm is inspired by the lifestyle of a family of birds called cuckoo. These birds' lifestyle, egg-laying features and breeding are the basis of the development of this optimization algorithm [29]. Like other evolutionary approaches, CS starts with an initial population (population of cuckoos). There are two types of the population of cuckoos in different societies: mature cuckoos and eggs. The basis of the algorithm is made by stimulating the obligate brood parasitism of some cuckoo species by laying their eggs in the nests of other host birds. Some host nest can keep direct difference. If an egg is discovered by the host bird as not its own, it will either throw the unknown egg away or simply abandon its nest and build a new nest elsewhere [9]. The principle of this species in the wild is the attempt to survive by any means. While competing for being survived, some of them are demised. The survived cuckoos immigrate to better areas and start reproducing and laying eggs [29].

In the process of cuckoo search algorithm, $n$ randomly chosen nests come into being, and the $i^{\text {th }}$ nest is set nest ${ }_{i}=$ $\left(x_{i 1}, x_{i 2}, \cdots x_{i d}\right)$, where $d$ is the dimension of the problem. The fitness of each nest can be got according to their own location information [7, 30]. The nests are updated according to Equation. (5): 


$$
\operatorname{nest}_{i}^{t+1}+\alpha \oplus \operatorname{lévy}(\lambda), \quad 1 \leq i \leq n
$$

where $\alpha$ is the step size according to the scale of the problem. The produce $\bigoplus$ is entry-wise multiplications. The random walk via Lévy flight is more efficient in exploring the search space as its step length is much longer in the long run [30]. The Levy flight essentially provides a random walk while the random step length is drawn from a Lévy distribution as shown in the Equation. (6):

$$
\text { Lévy u }=t^{-\lambda}, 1<\lambda \leq 3
$$

which has an infinite variance with an infinite mean. Each nest has a certain probability $\left(P_{a}\right)$ to be abandoned. If a nest is abandoned, a new nest will be created according to Equation. (7):

$$
n e s t_{i}^{t+1}=n e s t_{i}^{t}+r\left(\text { nest }_{j}^{t}-\text { nest }_{k}^{t}\right)
$$

where $r$ is a scale factor uniformly distributed between 0 and $1 ; n e s t_{j}^{t}$ and nest $t_{k}^{t}$ are other nests, randomly selected in $t^{\text {th }}$ generation [30]

\section{Cuckoo Search-based Neural Network (CSNN)}

In CSNN, CS is applied to optimize the initial weights and biases of backpropagation (BP) network. More specifically, the BP network is considered to be the objective function (fitness function), and the weights and biases are computed by the CS algorithm such as to maximize the objective function [31]. These optimal weights and biases used for the BP network are expected to be significantly superior to the basic BP network. The weight optimization problem and the size of the population determine the quality of the solution. In the first epoch, the best weights and biases are initialized with $\mathrm{CS}$ and then these weights are passed to the BPNN. The weights in BPNN are calculated and compared with the best solution in the backward direction. In the next cycle, CS will update the weights with the best possible solution and CS will continue searching the best weights either until the last cycle/epoch of the network is reached or the MSE is achieved [9].

The process of the BP network optimized by the CS is divided into three parts: determining BP network structure, obtaining the best weights and biases through CS, and predicting the output through the neural network. The elements in CSBP include initializing CS, determining fitness function, updating position operator, selecting operator, replacing operator, and eliminating operator in order to find the cuckoo individual with the best fitness. Based on the above analyses, the flowchart of the CSNN algorithm is shown in Figure 3.

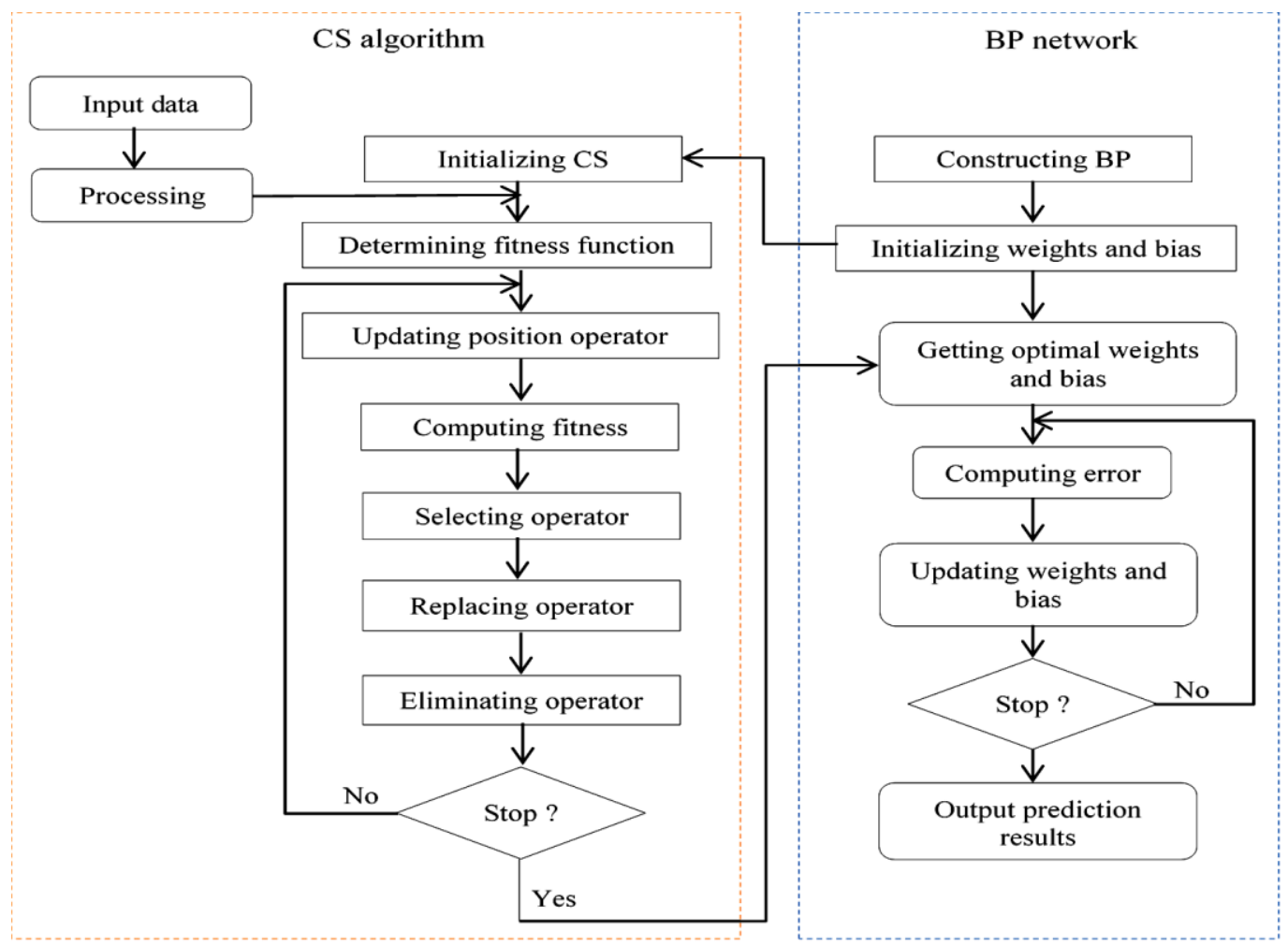

Figure 3. Flowchart for prediction with CSSN [31]. 
The flowchart above can be summarized by the following pseudo code:

Begin

Step 1. CS is initialized and passes the best weights to BP network

Step 2. Load the training data

Step 3. While MSE < stopping criteria

Initialize all cuckoo nests

Pass the cuckoo nests as weights to network

Feedforward neural network runs using the weights initialized with CS

Calculate the error backward

CS keeps on calculating the best possible weight at each epoch until the network is converged.

Step 4. End While

End

\section{RESULTS AND DISCUSSION}

This work focuses on the prediction of the fatigue life of various composite materials for the design of wind turbine blades, where all the studied materials are submitted to tension-tension fatigue loads. Data was collected from a variety of MSU/DOE fatigue database [10] with a stress ratio of $0.1\left(R=\sigma_{\min } / \sigma_{\max }=0.1\right)$. Once the CSNN has been shown to predict fatigue failure correctly under this condition, it is possible to extend the same technique to predict fatigue behavior under distinct stress ratio values.

For the lifetime prediction analysis of previous materials mentioned in Table 1, it is presumed that the fatigue life depends on the number of cycles to failure, minimum $\left(\sigma_{\min }\right)$ and maximum $\left(\sigma_{\max }\right)$ stresses applied to the specimen and the fiber orientation angle $(\theta)$. Other parameters that might affect the lifetime of materials, such as the microstructure and process parameters will not be considered in this paper. All specimens will be presumed to have similar characteristics in this respect.

\section{Adjustment of CSNN Topology Parameters}

As mentioned earlier, adequate choice of ANN parameters (inputs, structure...) is critical to obtaining appropriate outcomes. The network was trained and tested using experimental fatigue data from the materials shown in Table 1 . In order to achieve the optimum fatigue life prediction, the ANN architecture, training algorithms as well as the number of neurons per hidden layer were regarded.

Firstly, the performance of CS to optimize the weights and bias is tested with a discovery rate $p a=0.15$ and a small population size of 25 . For each prediction, trial is limited to 1000 epochs and minimum error is kept close to 0 . As intelligent algorithms always have some randomness, each run will generate different results. In order to get a typical statistical performance, 15 trials are run for each case. The network results are stored for each trial.

The CS is used to train the feedforward neural network to improve the convergence rate and learning process. The process of learning involves finding a set of weights to minimize the learning error. The goal in this algorithm is to minimize a learning error (cost function) calculated by using MSE (Equation. (8)). Each cuckoo individual contains all the weights and bias in BP network. According to the weights and bias in BP network, a certain BP network can be constructed. The parameters used for running the CS are mentioned in the paragraph above.

$$
j(W)=\frac{1}{2} \sum_{i=1}^{N}\left(\hat{y}^{i}-y^{i}\right)^{2}
$$

Here $j$ is the objective function, performance or cost function; $N$ is the number of nodes; $W$ is the weight matrix; $\hat{y}$ is the output obtained by the neural network; $y$ is the desired output.

The selection of these CSNN parameters plays an important role in optimization. A single CS parameter choice has a tremendous effect on the rate of convergence. For this paper, the optimal CS parameters are determined by trial and error experimentations. Optimal here refers to the set of CS parameters that yield the fastest neural network convergence.

Since the range of the number of cycles to failure varied between 170 and 5,000,000 cycles, training the networks to learn such a wide range will produce unacceptable and unbalanced modelling performance. This will happen as the CSNN attempts to minimize the global error for all input patterns. Minimizing the difference between network output and observed data for elevated stress cycle values would therefore lead in inaccurate results for patterns associated with reduced values of the number of failure cycles [26]. A more suitable method would be the range-normalized absolute difference (Equation. (9)) of the number of cycles, to reach a range between 0 and 1. For practical reasons, normalizing 
(or standardizing) the inputs in neural networks can make training faster and reduce the chances of being stuck in local minima.

$$
x_{n r m}=\frac{x_{i}-x_{\min }}{x_{\max }-x_{\min }}
$$

Here $x_{i}$ is the value we want to normalize; $x_{\max }$ and $x_{\min }$ are the maximum and minimum values of the number of cycles. The same normalization method was also conducted with the maximum applied tensile stress, which varied between 130 and $690 \mathrm{MPa}$. This is because the starting point of the optimization process of parameters begins with the calculation of the error as a difference between real and estimated values. Therefore, these two sets of values (number of cycles and max stress) have to have the same range. The Matlab (R2009b) Software was used to construct, train, and test the networks.

The NN used to predict the fatigue life of all materials investigated was a two-layer feedforward network. The normalized maximum tensile stress $\sigma_{\text {max }}$, ply-orientations (MD/UD fabrics) and the stress ratio $R$ were used as input parameters; while the normalized number of cycles $N$ was used as an output, with one computation neuron using a linear activation function. They communicate through a single hidden layer of ten (10) neurons, which uses a sigmoid activation function.

\section{Fatigue Life Prediction using CSNN}

The proposed modelling procedure is carried out with the help of 166 sets (data size) of experimental fatigue data. These represent a comparison between different materials based on different reinforcing fabrics (see Table 1), in terms of the number of cycles to failure. This can be withstood up to $690 \mathrm{MPa}$ as determined from the curve fits in Table 2 . Other measures of fatigue resistance such as the exponents of the S-N fit in Table 2 would show consistent trends.

One common problem that occurs during neural network training is "data overfitting", where the network tends to memorize the training examples without learning how to generalize to new situations. The default method on the Matlab software for improving generalization is called "early stopping" which consists in dividing the available training dataset into three subsets. From these 166 sets, only $60 \%$ of the samples are assigned to train the proposed CSNN model, $20 \%$ to the validation set and the remaining $20 \%$ are used for testing purpose.
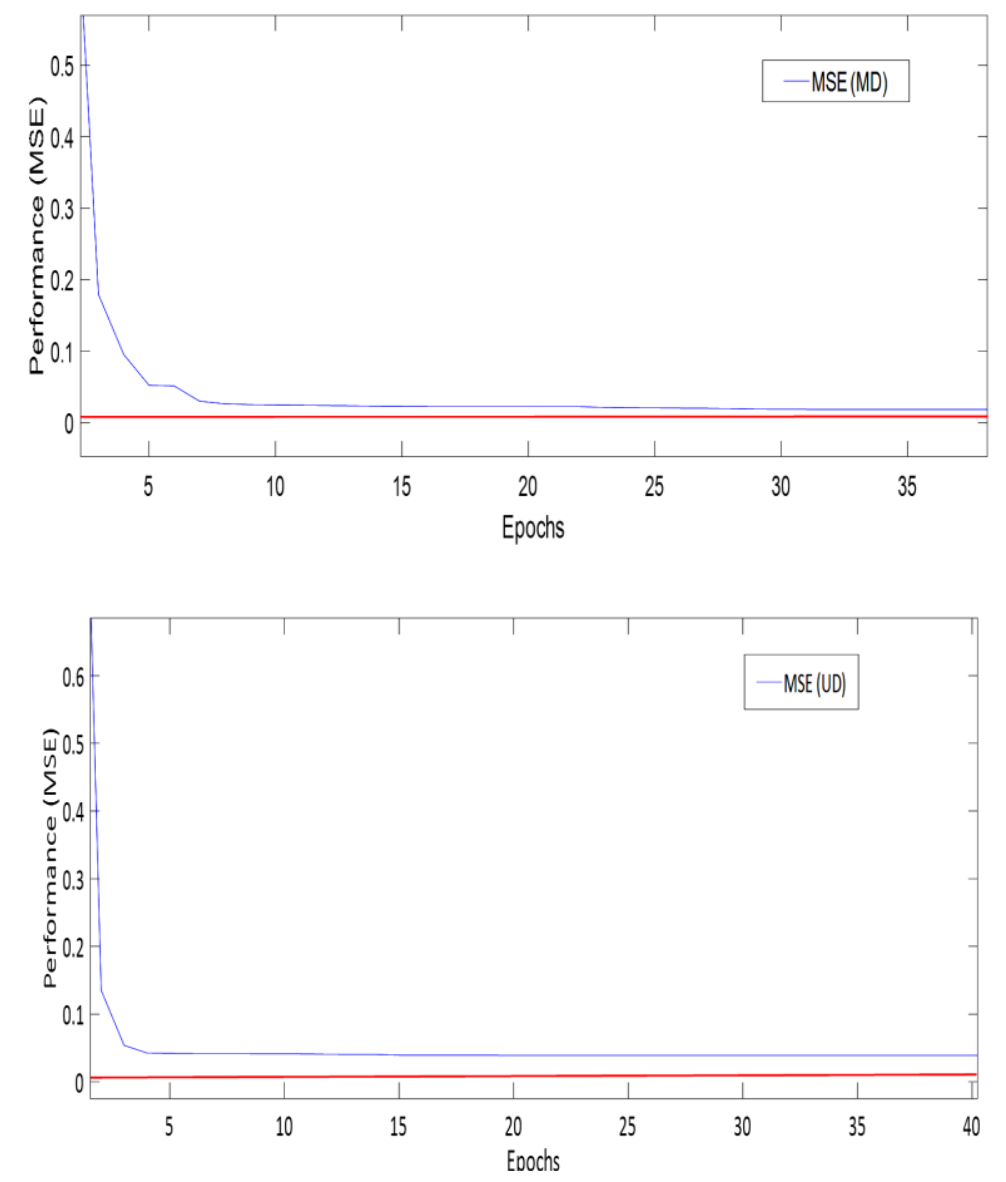

Figure 4. Variation of MSE for the training data according to the number of epochs.

Figure 4 shows typical variations of the MSE obtained using CSNN for MD/UD materials considered as a function of the number of epochs. This figure also shows a fast convergence of the MSE to global minima within 40 epochs. The 
used training proportion in the case of MD laminates is $60 \%$ of 78 samples, where the MSE decreases until it reaches the value of 0.0167 (equivalent to $1.67 \%$ ). While, for the UD laminates, the MSE reaches a value of 0.0380 (equivalent to $3.8 \%$ ), but this time, with a training proportion of $60 \%$ of 88 samples. These MSE values show the effectiveness of our CSNN topology, with ten hidden neurons.

Similarly, the Table 3 describes the CPU time, number of epochs and the MSE of the CSNN model. From this table, we can see that the CSNN method performs well on MD/UD datasets. The CSNN converges to global minima in 8.48 second of CPU time, with an average accuracy of $86 \%$ for the MD laminates.

Table 3. CPU time, epochs and MSE for MD/UD training set.

\begin{tabular}{ccc}
\hline MD/UD materials & MD Laminates & UD Laminates \\
\hline CPU Time (sec.) & 8.486 & 8.678 \\
Epochs & 35 & 40 \\
MSE & 0.0167 & 0.0380 \\
Accuracy $(\%)$ & 86.472 & 79.832 \\
\hline
\end{tabular}

Both experimental and predicted results obtained with CSNN are compared in Figures. 5-9 for the materials considered and indicate the relation between the maximum applied stress and the number of cycles to failure. The figures show typical fatigue life predictions of glass-fiber/epoxy, polyester and vinyl esters materials using CSNN model with ten hidden neurons. Each figure presents two plots for different ply orientations. As well as, the figures show a shift between the experiments and the predicted values along the stress axis $\sigma$ for all laminates studied with $\left[\left( \pm 45^{\circ}\right)_{2} /(0)_{2}\right]_{s}$ and $[0]_{2}$ fiber orientations. The nonlinear modeling obtained by CSNN can be utilized to predict the fatigue life for these fiber orientations, for any materials at the desired loading conditions.

Based on actual data in Table 2, the multidirectional glass laminates $\left[\left( \pm 45^{\circ}\right)_{2} /(0)_{2}\right]_{s}$ under tensile fatigue loading $(R=0.1)$ are very sensitive to the fiber orientation and small details in fabric construction. On the other hand, the laminates based on unifabric $[0]_{2}$ show good fatigue performance, in terms of fatigue exponent and strain capacity at $10^{6}$ cycles (equivalent to 1 after normalization).
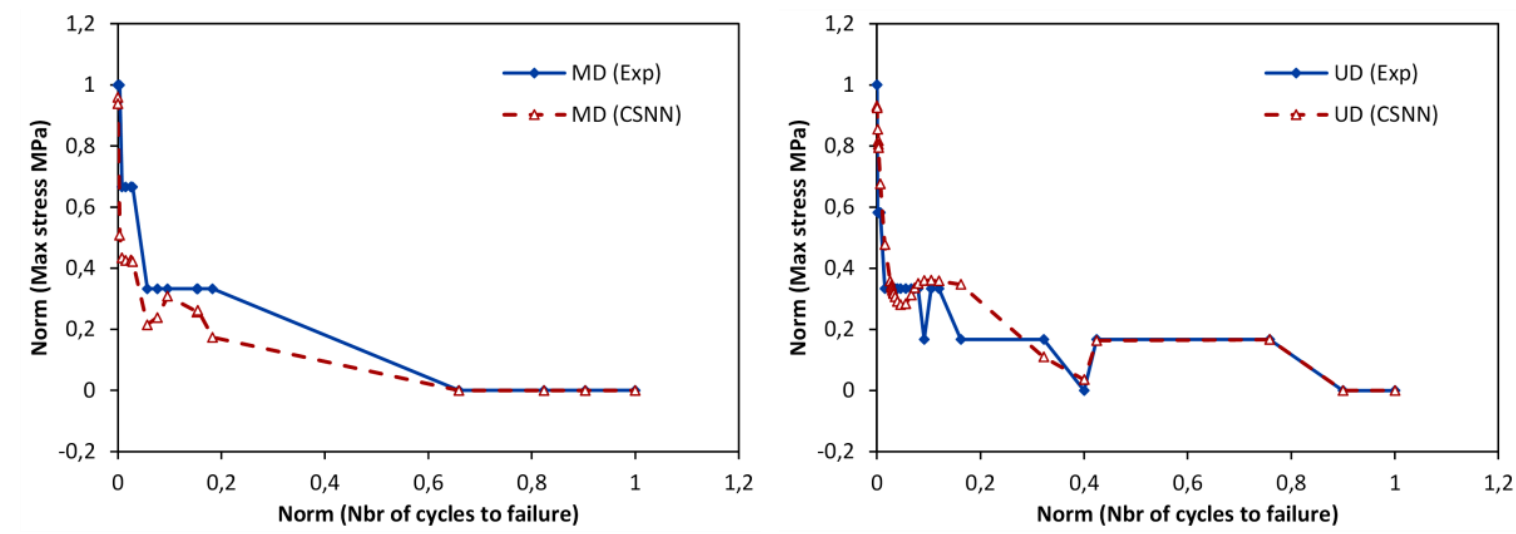

Figure 5. Fatigue life prediction of PPG1200-EP1 glass-fiber/epoxy for multidirectional $\left[\left( \pm \mathbf{4 5} 5^{\circ}\right)_{2} /(\mathbf{0})_{2}\right]_{s}$ and unidirectional $[\mathbf{0}]_{2}$ laminates.
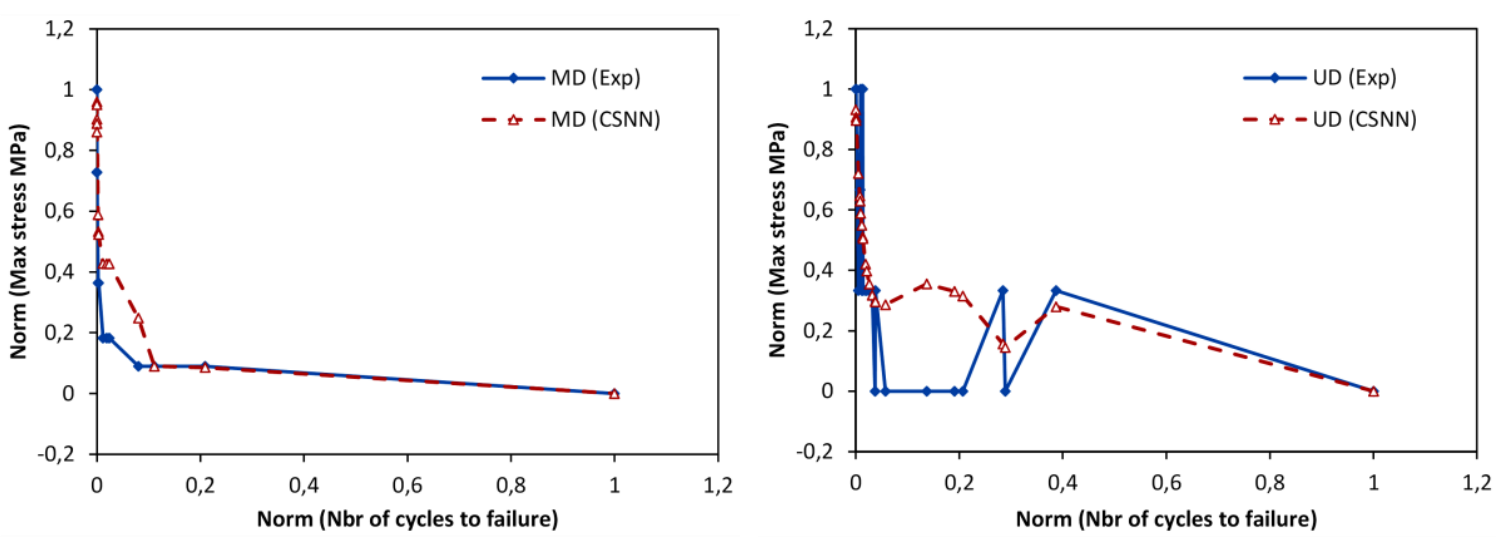

Figure 6. Fatigue life prediction of PPG1200-UP5 glass-fiber/polyester for multidirectional $\left[\left( \pm \mathbf{4 5} 5^{\circ}\right)_{2} /(\mathbf{0})_{2}\right]_{s}$ and unidirectional $[\mathbf{0}]_{2}$ laminates. 

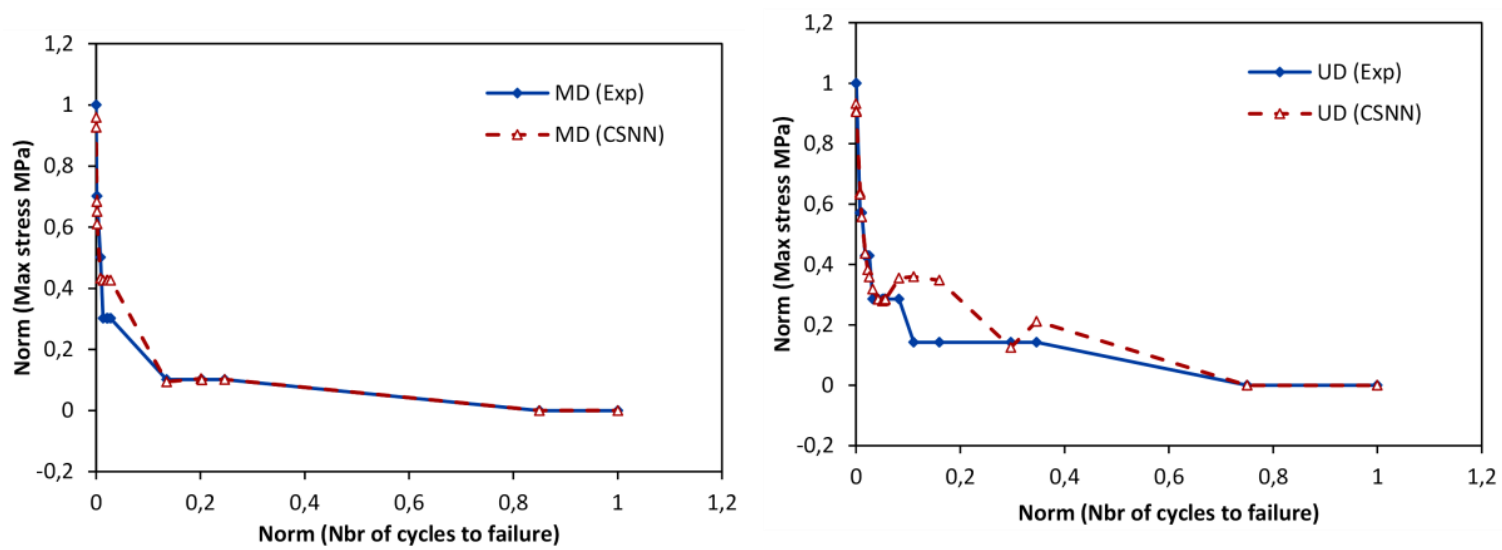

Figure 7. Fatigue life prediction of PPG1200-VE4 glass-fiber/vinylester for multidirectional $\left[( \pm \mathbf{4 5})_{2} /(\mathbf{0})_{2}\right]_{s}$ and unidirectional $[\mathbf{0}]_{2}$ laminates.
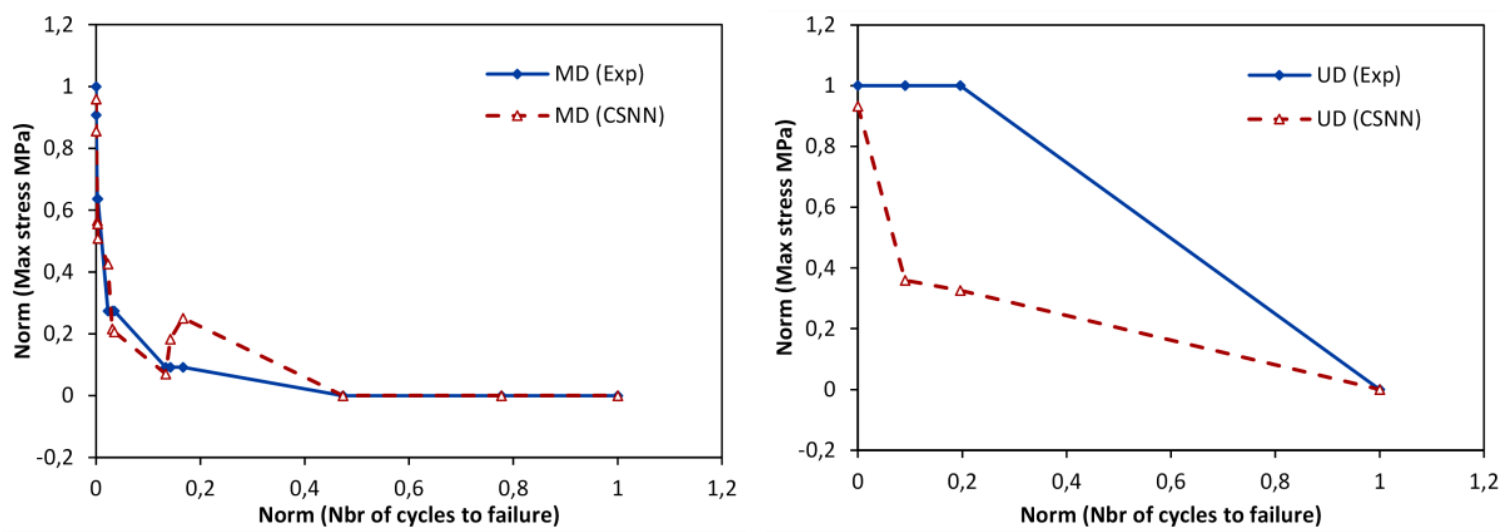

Figure 8. Fatigue life prediction of PPG1200-VE5 glass-fiber/vinylester for multidirectional $\left[( \pm \mathbf{4 5})_{2} /(\mathbf{0})_{2}\right]_{s}$ and unidirectional $[\mathbf{0}]_{2}$ laminates.

The CSNN predictions were obtained using a variety of architectures and topologies with different numbers of hidden neurons. The choice stops when the network converges or reaches a global minimum already predefined as objective function (MSE). Table 4 shows the values of the root mean square error RMSE obtained when predicting the fatigue life of each MD/UD material.
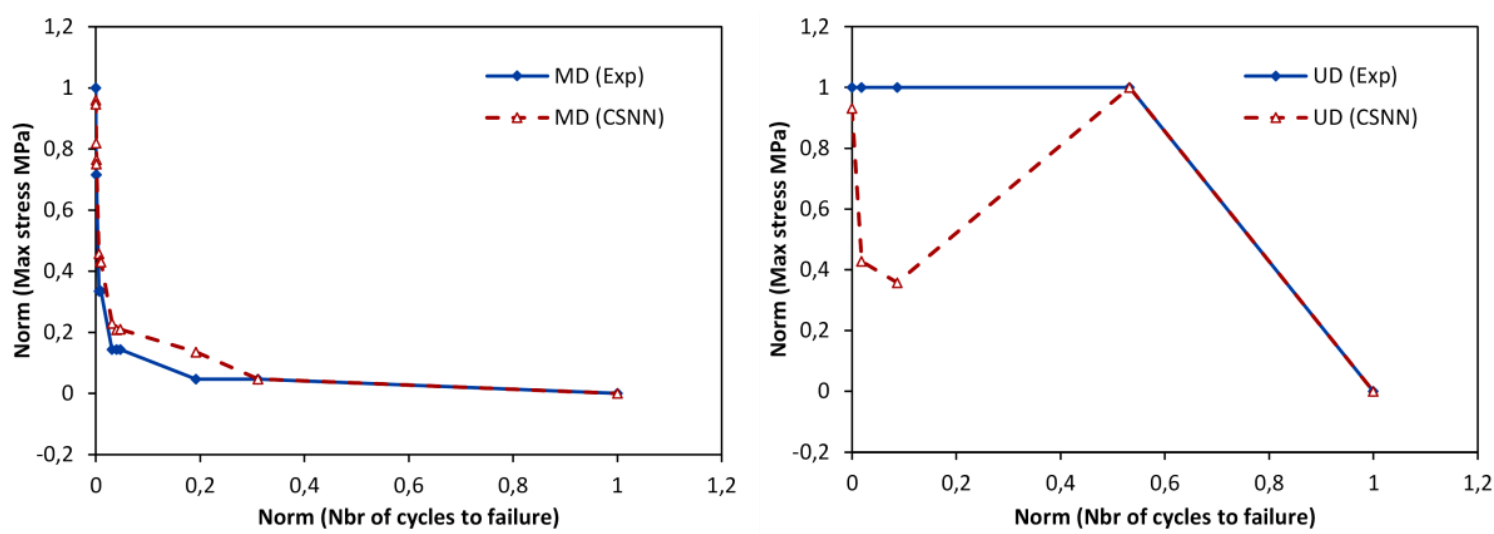

Figure 9. Fatigue life prediction of PPG1200-VE6 glass-fiber/vinylester for multidirectional $\left[( \pm \mathbf{4 5})_{2} /(\mathbf{0})_{2}\right]_{s}$ and unidirectional $[\mathbf{0}]_{2}$ laminates. 
Table 4. RMSE values for all MD/UD materials investigated.

\begin{tabular}{lcc}
\hline \multirow{2}{*}{ Materials } & \multicolumn{2}{c}{ RMSE (\%) } \\
\cline { 2 - 3 } & MD Laminates & UD Laminates \\
\hline PPG1200-EP1 glass-fiber/epoxy & 18.49 & 10.22 \\
PPG1200-UP5 glass-fiber/polyester & 16.05 & 22.66 \\
PPG1200-VE4 glass-fiber/vinylester & 7.30 & 8.09 \\
PPG1200-VE5 glass-fiber/vinylester & 8.34 & 46.63 \\
PPG1200-VE6 glass-fiber/vinylester & 7.40 & 38.58 \\
\hline
\end{tabular}

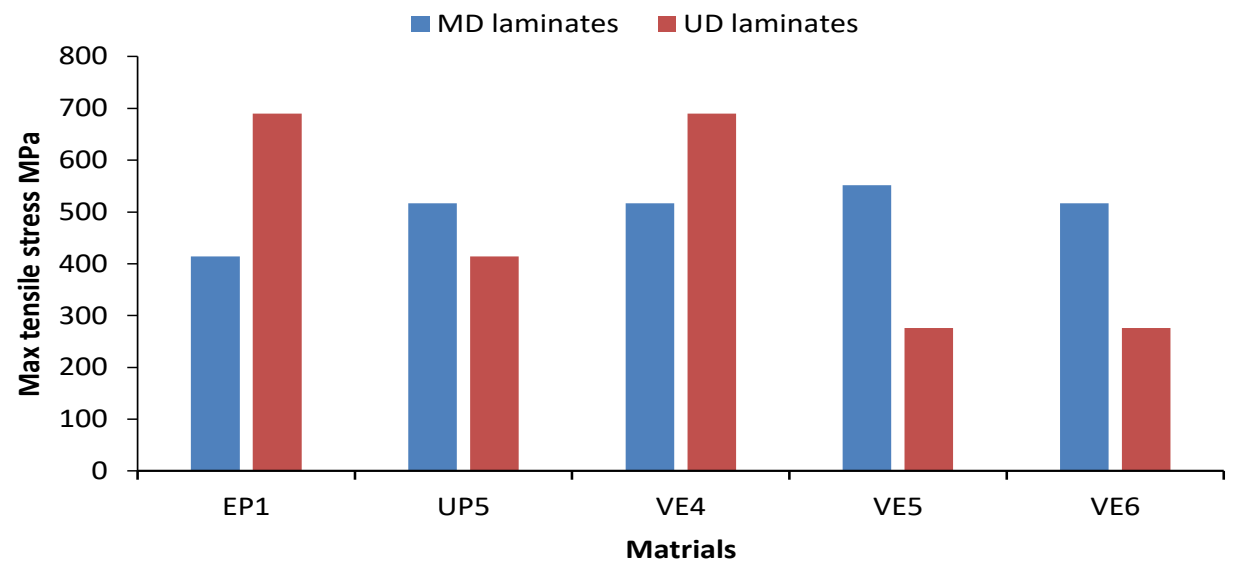

Figure 10. Comparison of fatigue strength of all materials investigated.

As it is clear from all these figures, the predicted and experimental results are very close, except in the last two figures (Figure 8 and Figure 9). This is due to insufficient data, particularly with unidirectional laminates. Therefore, of the 166 sets, only 8 are used for the experimental fatigue tests of unidirectional VE5 and VE6 as well as for the training of our CSNN model. The RMSE obtained for these two materials (Figure 8 and Figure9) were found to be $46.63 \%$ and $38.58 \%$ respectively, which means the large variation between experimental and predicted values.

To make this result more evident, we needed to quantify the comparison by a bar chart. In Figure 10, both MD and UD laminates are compared in terms of maximum tensile strength. The values mentioned are taken directly from the source database in MPa, without normalization. All materials showed a disparity in the values of the tensile fatigue strength. The MD laminates with $\left[\left( \pm 45^{\circ}\right)_{2} /(0)_{2}\right]_{s}$ fiber orientation present a consistency in their values (between 400 $\mathrm{MPa}$ and $500 \mathrm{MPa}$ ). The decrease in the fatigue strength is very significant for the PPG1200-VE5 glass-fiber/vinylester and PPG1200-VE6 glass-fiber/vinylester, especially with the unifabric $[0]_{2}$ laminates. From the same figure, PPG1200EP1 glass-fiber/epoxy and PPG1200-VE4 glass-fiber/vinylester showed a higher fatigue strength compared to other materials.

Comparing with the results obtained with PSO-ANN in [11], CSNN gave us better fatigue life predictions, faster computation, more accuracy and above all, a high convergence rate at the true global minimum.

\section{CONCLUSIONS}

Different materials based on different reinforcing fabrics and resins are compared in terms of fatigue life. The combination cuckoo search-based artificial neural network (CSNN) has been used to predict the fatigue life of these composite materials, which are all intended for the design of wind turbine blades. The CSNN model applied in this paper highlight the following conclusions:

- The limitations of traditional fatigue prediction methods based on human experience; hence the need to use neural networks that can provide excellent results.

- The prediction of the fatigue behavior of wind turbine blades can greatly help to improve the quality of their prevention.

- Reduced lab testing time, which also reduces the cost.

- Correct and adjust experimental tests by linear regression of predicted values.

- Optimize the MSE between experimental and predicted results.

- The size and quality of the database have a major influence on the learning of neural networks. For this reason, the choice of a database is sometimes the only parameter deciding the quality of learning.

- The effects of ANN architecture, training algorithm as well as number of neurons per hidden layer were considered to obtain the optimum fatigue life prediction. 
- A supervised network trained by a metaheuristic algorithm gives results with an accuracy that had each of them separately.

- CSNN is a very good combination for applications that require fast learning/convergence.

- The right selection of CS algorithm parameters plays a key role in optimization.

In addition to creating a model that can predict the fatigue life of different MD/UD composite materials. The results obtained by the CSNN approach allowed us to suggest certain types of composite materials, from a lifetime and strength standpoint, for an eventual design of wind turbine blades, taking into consideration the following points:

- The materials based on UD fabric retain good tensile fatigue life compared to those based on MD fabric

- Glass-fiber/epoxy (EP1) and glass-fiber/vinylester (VE4) are least sensitive to the fiber orientation among the five materials investigated

As perspective, further research is needed to determine the possibility of finding unique combinations that always result in the best fatigue life predictions, as well as to improve the ANN's accuracy by using other optimization algorithms in their learning.

\section{ACKNOWLEDGMENTS}

The Authors would like to thank Sandia National Laboratories (SNL) and Montana State University (MSU) for the dataset used in this paper.

\section{REFERENCES}

[1] Attaf B. Designing composite wind turbine blades from cradle to cradle. In Recent advances in composite materials for wind turbine blades; Attaf, B., Ed.; The world academic publishing, Hong Kong. 2013;2-24.

[2] Nijssen RPL. Fatigue life prediction and strength degradation of wind turbine rotor blade composites. PhD Thesis. Delft University the Netherlands, Delft. 2006.

[3] Mishnaevsky Jr L, Branner K, Petersen HN, Beauson J, McGugan M, F. Sørensen B. Materials for wind turbine blades: An overview. Materials. 2017;10:1-24.

[4] Beauson J, Madsen B, Toncelli C, Brøndsted P, Bech JI. Recycling of shredded composites from wind turbine blades in new thermoset polymer composites. Compos. Part A. 2016;90:390-99.

[5] Mandell JF, Samborsky DD, Miller DA. Effects of resin and reinforcement variations on fatigue resistance of wind turbine blades. In Advances in wind turbine blade and design. Brondsted P, Nijssen RPL, Eds., Woodhead Publishing Limited, UK. 2013;210-50.

[6] Al-Assadi M, El Kadi H, Deiab IM. Using artificial neural networks to predict the fatigue life of different composite materials including the stress ratio effect. Appl. Compos. Mater. 2011;18:297-309.

[7] Yang XS, Deb S. Cuckoo search via Lévy flights. World Congress on Nature \& Biologically Inspired Computing. India. 2009.

[8] Yang XS. Nature-Inspired Metaheuristic Algorithms Second Edition. Luniver Press, UK, 2010.

[9] Nawi NM, Khan A, Rehman MZ. A new back-propagation neural network optimized with cuckoo search algorithm. International Conference on Computational Science and Its Applications. Ho Chi Minh, 2013.

[10] SNL/MSU/DOE Composite Material Fatigue Database. Mechanical properties of composite materials for wind turbine blades. Montana State University-Bozeman, Version 25.0, Available online: http://energy.sandia.gov/ (accessed on 20 October 2016).

[11] Ziane K, Zebirate S, Zaitri A. Particle swarm optimization-based neural network for predicting fatigue strength in composite laminates of wind turbine blades. Composites: Mechanics, Computations, Applications: An International Journal. 2015;6:32138.

[12] Ziane K, Zebirate S, Zaitri A. Fatigue strength prediction in composite materials of wind turbine blades under dry-wet conditions: An artificial neural network approach. Wind Engineering. 2016;40:189-98.

[13] Al-Assadi M, El-Kadi H, Deiab IM. Predicting the fatigue life of different composite materials using artificial neural networks. Appl. Compos. Mater. 2010;17:1-14.

[14] Mathur S, Gope P, Sharma J. Prediction of fatigue lives of composites material by artificial neural network. In Proceedings of the SEM 2007 Annual Conference and Exposition, Springfield, Massachusetts, USA. 2007; Paper No. 260.

[15] Jiménez AA, Gómez Muñoz CQ, García Márquez FP. Machine learning for wind turbine blades maintenance management. Energies. 2017;11:1-16.

[16] Liu X, Liu Z, Liang Z, Zhu S, Correia JAFO, De Jesus AMP. PSO-BP Neural Network-Based Strain Prediction of Wind Turbine Blades. Materials. 2019;12:225-34.

[17] Mandell JF, Samborsky DD, Miller DA. Analysis of SNL/MSU/DOE Fatigue Database Trends for Wind Turbine Blade Materials, 2010-2015. Research Report No. SAND SAND2016-1441, Sandia National Laboratories. 2016.

[18] Samborsky DD, Agastra P, Mandell JF. Fatigue trends for wind blade infusion resins and fabrics. Proc. 51 AIAA/ASME/ASCE/AHS/ASC Structures, Structural Dynamics, and Materials Conference, Florida. 2010.

[19] Agastra P, Samborsky DD, Mandell JF. Fatigue resistance of fiberglass laminates at thick material transitions. Proc. 50 AIAA/ASME/ASCE/AHS/ASC Structures, Structural Dynamics and Materials Conference, California. 2009. 
[20] Mandell JF, Samborsky DD, Agastra P, Sears AT, Wilson T.J. Analysis of SNL/MSU/DOE Fatigue Database Trends for Wind Turbine Blade Materials. Research Report No. SAND2010-7052, Sandia National Laboratories. 2010.

[21] Ziane K. Analyse, évaluation et réduction des risques d'un parc éolien. PhD Dissertation, Université d'Oran 2 Mohamed Ben Ahmed, Oran. 2017.

[22] Svozil D, KvasniEka V, Pospichal J. Introduction to multi-layer feedforward neural networks. Chemometrics and Intelligent Laboratory Systems. 1997;39:43-62.

[23] Rath AK, Das HC, Parhi DR, Kumar PB. Application of artificial neural network for control and navigation of humanoid robot. Journal of Mechanical Engineering and Sciences. 2018;12:3529-38.

[24] Hanief M, Wani MF. Artificial neural network and regression-based models for prediction of surface roughness during turning of red brass (C23000). Journal of Mechanical Engineering and Sciences. 2016;10:1835-45.

[25] Mohanty JR, Verma BB, Parhi DRK, Ray PK. Application of artificial neural network for predicting fatigue crack propagation life of aluminum alloys. Archives of Computational Materials Science and Surface Engineering. 2009;1:133-38.

[26] Al-Assaf Y, El-Kadi H. Fatigue life prediction of unidirectional glass fiber/epoxy composite laminae using neural networks. Compos. Struct. 2001;53:65-71.

[27] Vassilopoulos AP, Georgopoulos EF, Dionysopoulos V. Artificial neural networks in spectrum fatigue life prediction of composite materials. International Journal of Fatigue. 2007;29:20-29.

[28] Nawi NM, Khan A, Rehman MZ. A New Cuckoo Search Based Levenberg-Marquardt (CSLM) Algorithm. International Conference on Computational Science and Its Applications, Ho Chi Minh. 2013.

[29] Jafari S, Bozorg-Haddad O, Chu X. Cuckoo Optimization Algorithm. In Advanced Optimization by Nature-Inspired Algorithms; Bozorg-Haddad, O., Ed.; Springer: Singapore. 2018;39-49.

[30] Ding J, He X, Jiang B, Wu Y. Parameter identification for area-specific resistance of direct methanol fuel cell using cuckoo search algorithm. In Bio-Inspired Computing - Theories and Applications. Gong M, Linqiang P, Tao S, Tang K, Zhang X, Eds.; Springer: Heidelberg, Germany. 2015;107-12.

[31] Yi J, Xu W, Chen Y. Novel back propagation optimization by cuckoo search algorithm. The Scientific World Journal. 2014;18. 